\title{
Theoretical Model and Experimental Validation of the estimated proportions of common and independent input to motor neurons *
}

\author{
A. Margherita Castronovo, Member, IEEE, Francesco Negro, Member, IEEE, \\ and Dario Farina, Member, IEEE
}

\begin{abstract}
Motor neurons in the spinal cord receive synaptic input that comprises common and independent components. The part of synaptic input that is common to all motor neurons is the one regulating the production of force. Therefore, its quantification is important to assess the strategy used by Central Nervous System (CNS) to control and regulate movements, especially in physiological conditions such as fatigue. In this study we present and validate a method to estimate the ratio between strengths of common and independent inputs to motor neurons and we apply this method to investigate its changes during fatigue. By means of coherence analysis we estimated the level of correlation between motor unit spike trains at the beginning and at the end of fatiguing contractions of the Tibialis Anterior muscle at three different force targets. Combining theoretical modeling and experimental data we estimated the strength of the common synaptic input with respect to the independent one. We observed a consistent increase in the proportion of the shared input to motor neurons during fatigue. This may be interpreted as a strategy used by the CNS to counteract the occurrence of fatigue and the concurrent decrease of generated force.
\end{abstract}

\section{INTRODUCTION}

The estimation of the various components of the synaptic input delivered to $\alpha$-motor neurons, is one of the major issues in understanding how the Central Nervous System (CNS) controls and regulates movements. Motor neurons in the spinal cord receive, in fact, both common and independent inputs from spinal and supra-spinal regions [1]. In particular, the common component of the drive has been shown to be the only component necessary for force control [2] and thus, its quantification may be useful for better understanding of complex physiological phenomena such as fatigue. The presence of common synaptic input to motor neurons may be either an efficient way to control motor performance [3][4] or it may disrupt it [5][6]. The common part of the synaptic input to motor neurons induces correlation between output spike trains which is thought to arise at a pre-synaptic level [7]. This phenomenon is often referred to as "short term synchronization" [8] and can be estimated either in the temporal domain using cross-correlation techniques [9] or in

*Research supported by ERC Advanced Grant “DEMOVE”(no 267888) A. Margherita Castronovo, Francesco Negro and Dario Farina are with the Department of Neurorehabilitation Engineering, Bernstein Center for Computational Neuroscience, Bernstein Focus Neurotechnology, the spectral domain by computing coherence functions [10]. The low frequency range in correlation (up to $5 \mathrm{~Hz}$ ) reflects the usually termed 'common drive' to motor neurons [11] while the range 5-12 is related to the generation of relatively slow movements and tremorogenic oscillations [12]. Higher frequency bands are thought to mainly the interaction between the motor cortex and the spinal pathways [13]. We have recently shown that the proportion of the common synaptic input tends to increase with force, but there is still a debate on changes due to adaptations of the system to fatigue [14]. Some investigators have reported an enhancement of coherence just in the beta [15] or both in beta and gamma bands [16] during fatigue in the Tibialis Anterior [15] and hand muscles [16] but other studies have shown an opposite behavior [17][18].

The aim of this work is to investigate the changes in the proportion of the common synaptic input to motor neurons during fatiguing contractions of the Tibialis Anterior muscle. For this purpose, we first introduce a theoretical model of coherence as a function of the number of motor unit spike trains. A parameter expressing the ratio between common and independent components of the synaptic input is introduced and used as a novel physiological measure of the common input strength [19]. The theoretical derivations will be then applied to experimentally recorded motor unit spike trains obtained from the Tibialis Anterior muscle of three healthy subjects during sustained fatiguing contractions. Results about the changes of the new synchronization index during fatigue are then presented and discussed.

\section{METHODS}

\section{A. Theoretical modeling}

The new indicator of the strength of common synaptic input was estimated by modelling a population of $n$ motor neurons and by computing the coherence function on the output spike trains. Coherence is a mathematical measure of correlation between two signals in the frequency domain and its values span the range [0-1], where 0 indicates no correlation at all and 1 means a perfect correlation. It is defined as:

Universitätsmedizin Göttingen (UMG), Georg-August-Universität, von Siebold Str. 6, 37075 Göttingen, Germany (email: \{margherita.castronovo, negro.francesco, dario.farina\} @ bccn.uni-goettingen.de). 


$$
C_{x y}(f)=\frac{\left|P_{x y}(f)\right|^{2}}{\left|P_{x x}(f)\right|\left|P_{y y}(f)\right|}
$$

where $P_{x y}(f)$ is the cross-spectral density and $P_{x x}(f)$ and $P_{y y}(f)$ the auto-spectral densities of the two signals, respectively. When considering a population of $n$ motor neurons, each producing an output discharge pattern, the coherence between the sums of two groups of $n$ spike trains is theoretically approximated as:

$$
C(f)=\frac{\left|n^{2} A\right|^{2}}{\left[n B+n^{2} A\right]^{2}}
$$

where, $A$ represents the power of the common synaptic input in the frequency range of interest and $B$ is the power of independent synaptic noise. For this approximation, we assumed that (i) each of the $n$ motor neurons receives a common and an independent input, (ii) the input-output relation of the motor neuron is approximately linear [20], (iii) the frequency band of the common stimulus is lower than the average discharge rate of the $n$ motor neurons, and (iv) the susceptibility of the motor neurons is constant in the frequency band of interest. In Eq. 2, A is obtained as the product of the spectrum of the stimulus modulated by the response function of the motor neuron to the stimulus, while $B$ is the power spectrum of the output spike train when the motor neuron receives only independent noise as input. It is worth noticing that, according to Sharafi et al., the independent synaptic noise sums linearly with $n$ (i.e. the number of motor neurons), while, the common synaptic input sum as $n^{2}$ [20]. Consequently, the common component of the synaptic input increases quadratically with the number of motor unit in the motor neuron pool.

Coherence, as expressed by Eq. 2, is a monotonic function increasing with the number of spike trains $(n)$ and with the variation of the common input with respect to the independent one [21][22]. This rising rate becomes faster as soon as the independent component is canceled. The upper limit of this function is 1 . The ratio $\gamma$ between the power of the common synaptic input and the power of the independent input is given by the ratio between $A$ and $B$ :

$$
\gamma=\sqrt{\frac{A}{B}}
$$

From a physiological point of view, $\gamma$ represents the proportion of the common synaptic input with respect to the independent one and thus can be used as an estimator of the strength of the common synaptic input to motor neurons.

\section{B. Experimental Validation}

The theory presented has been experimentally validated using high density surface electromyographic recordings obtained during sustained fatiguing contractions.

Participants - Three healthy men (age: $32 \pm 0.5$ ) performed sustained contractions of the Tibialis Anterior muscle at three different force levels. The experimental protocol was approved by the local ethical committee (approval no. 04/12/11) and an informed consent was signed by all the participants.

Experimental Protocol - Participants were seated on a commercial dynamometer device (Biodex Multi Joint System 3, Biodex Medical systems, Shirley, NY) with the leg fully extended and the foot attached to a force transducer by means of Velcro straps. Participants were instructed to dorsiflex the ankle by tracking a visual feedback of the force level to be maintained. Each contraction was then sustained until the subjects reached the task failure, identified as the time instant when the force produced dropped of $10 \%$ MVC with respect to the target force for more than $2 \mathrm{~s}$. The three contractions were performed on separate days and were randomized in order.

EMG Acquisition and Processing - High density surface EMG signals (sEMG) were recorded using a 64 channels array (8-mm inter-electrode distance; OT Bioelettronica, Torino, Italy), mounted on the surface of the Tibialis Anterior muscle with an adhesive foam. The sEMG signals were amplified (EMG-USB, LISiN - OT Bioelettronica, Torino, Italy), sampled at 2048 samples/s, and then digitized with a 12 bit A/D converter. Only the first ('beginning') and the last $30 \mathrm{~s}$ (before task failure, 'fatigue') of the electromyographic recordings were decomposed into motor unit spike trains using the CKC decomposition algorithm [23]. The correlation between identified motor unit spike trains (MUST) was estimated by computing coherence between the unfiltered composite spike trains (CST), obtained as the sum of the trains of discharge times in the decomposed time interval [21][24]. For both beginning and fatigue conditions, the CST was obtained by varying the number of MUST between 1 and the maximum number possible, i.e. half of the number of spike trains available for that particular subject and contraction level. For each number of MUST in the CST, we computed 100 realizations of coherence. A single estimate was then obtained by averaging coherence across all realizations and integrating in the frequency range [1-5] $\mathrm{Hz}$, i.e. the bandwidth of the common synaptic input [2].

Statistics - The coherence values obtained for each subject and each contraction level as a function of the number of spike trains in the CST were fitted with Eq. 2. The values of the A and B parameters were then used to calculate $\gamma$ according to Eq. 3 for both conditions (beginning and fatigue). $\gamma$ values are reported for each condition and contraction level as mean \pm standard deviation.

\section{RESULTS}

We decomposed a total number of 312 motor unit spike trains (150 for the beginning and 162 for the fatigue condition).

The average maximum number of motor units used to compute the CST was different among contraction forces: $9 \pm 2$ for the beginning and $10 \pm 3$ for the fatigue at $20 \% \mathrm{MVC}, 7 \pm 2$ 
for the beginning and $7 \pm 1$ for the fatigue at $50 \% \mathrm{MVC}$ and lastly $5 \pm 1$ for the beginning and $6 \pm 1$ for the fatigue at $75 \%$ MVC. In Figure 1, coherence profiles obtained by varying the number of MUST in the CST are depicted.

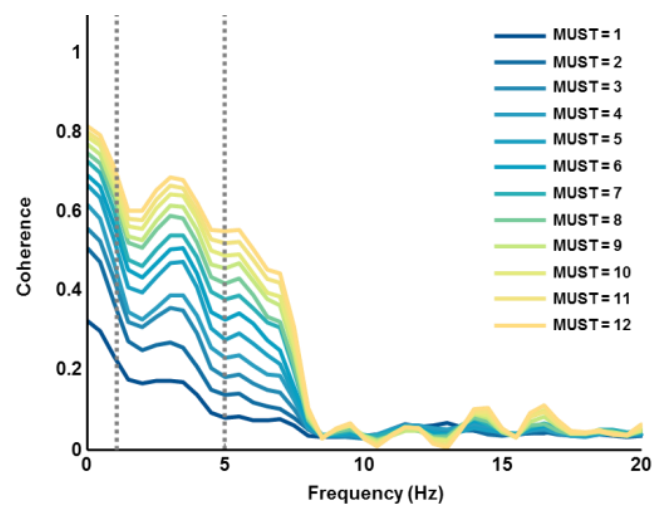

Figure 1. Coherence profiles obtained by varying the number of motor unit spike trains in the CST are depicted for the first 30 s of a $20 \%$ MVC contraction. Two vertical dotted grey lines have been placed to mark the frequency range in which the coherence values were averaged $(1-5 \mathrm{~Hz})$.

The coherence estimates integrated in the 1-5 Hz range were then fitted using the curve expressed by Eq. 2 .

Figure 2 shows an example of the fitting of the coherence values for the $20 \% \mathrm{MVC}$ force level. The coherence estimates obtained by averaging across 100 realizations and by varying the number of spike trains in the CST are depicted as mean \pm standard deviation in blue and yellow for the beginning and the fatigue condition, respectively. As soon as the number of spike trains used to compute the CST increases, the variance of the coherence estimation tends to decrease, being almost equal to zero for the last point in both conditions. The squared 2-norm of the residuals of the fitting decreased for the fatigue curve at both $20 \%$ and $50 \%$ MVC. Values are reported in Table I.

TABLE I.

SQUARED 2-NORM OF THE RESIDUALS OF THE NONLINEAR FITTING OF THE COMPUTED COHERENCE VALUES, AVERAGED ACROSS ALL SUBJECTS FOR EACH FORCE LEVEL

\begin{tabular}{ccc}
\hline \hline \multicolumn{3}{c}{$\begin{array}{c}\text { SQUARED 2-NORM OF RESIDUALS OF } \\
\text { NON-LINEAR FITTING }\end{array}$} \\
\hline \hline FORCE LEVEL & BEGINNING & FATIGUE \\
\hline $20 \% \mathrm{MVC}$ & $0.02 \pm 0.01$ & $0.004 \pm 0.001$ \\
$50 \% \mathrm{MVC}$ & $0.01 \pm 0.01$ & $0.003 \pm 0.001$ \\
$75 \% \mathrm{MVC}$ & $0.01 \pm 0.004$ & $0.01 \pm 0.003$ \\
\hline \hline
\end{tabular}

The example reported in Figure 2 highlights how during fatigue the coherence values increases with respect to the beginning for all numbers of MUST, due to an increase of the proportion of common input with respect to the independent one.

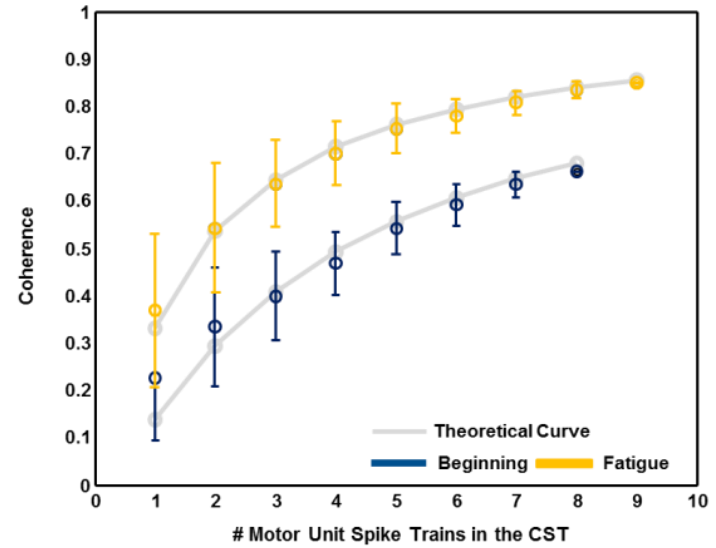

Figure 2. Example of fitting of the coherence values estimated at the beginning and at the end of a 50\%MVC fatiguing contraction with the curve of Eq. 2. The theoretical curve is depicted in grey for both conditions. It is possibile to notice how the experimental values fit the theoretical curve and how the standard deviation decreases as soon as the number of motor unit spike trains in the CST increases.

This behavior has also been confirmed by the increased values reported for $\gamma$. In fact, $\gamma$ changed from $0.52 \pm 0.1$ to $0.71 \pm 0.1$ and from $0.61 \pm 0.1$ to $1.13 \pm 0.02$, for $20 \%$ and $50 \% \mathrm{MVC}$, respectively. As mentioned before for the squared 2-norm of the residuals, we did not report any substantial variations in $\gamma$ values during fatigue for $75 \%$ MVC (Figure 3).

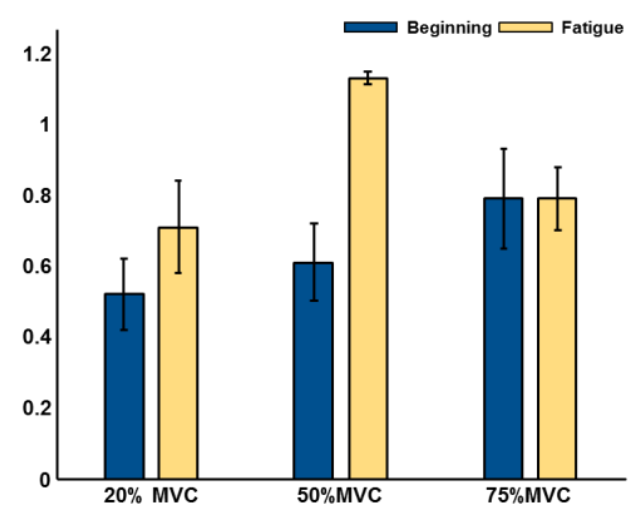

Figure 3. Average and standard deviation of $\gamma$ values across all subjects and for all contraction levels. $\gamma$ represents the ratio between the power of the common synaptic input and the power of the synaptic noise in the frequency range 1-5 Hz. Therefore it can be used as an indicator of the strenght of the common input with respect to the independent one. During fatigue $\gamma$ values increase for force levels such as $20 \%$ and $50 \%$ MVC.

\section{DISCUSSION}

We investigated the changes in the strength of common synaptic input to motor neurons with respect to the independent one during fatiguing contractions at different force targets. We have shown that in conjunction with the occurrence of task failure the common part of the synaptic input increases its proportion. When fatigue occurs the Central 
Nervous System intensifies the common component of the synaptic input shared across motor neurons. It has also been demonstrated that motor neurons are regulated by a balance between the excitatory and inhibitory synaptic inputs. However, when the level of net excitation increases, the conductance and the fluctuations in the membrane potentials do the same, resulting in an overall increase of the synaptic noise [25]. With our results we show that in conditions of increased excitation to motor neurons due to fatigue, the proportion of shared input increases even more. This is evident for force levels such as $20 \%$ and 50\%MVC. We also considered contractions at $75 \% \mathrm{MVC}$, but for this force target we did not report any change in the relative contribution of the common synaptic input.

The strength of the common synaptic input was estimated with a novel parameter, i.e. $\gamma$, which is the ratio between the power of the common synaptic input and the power of independent synaptic noise in the frequency range $1-5 \mathrm{~Hz}$. This parameter was estimated by using theoretical modelling to define a function expressing the changes in coherence with respect to the number of motor unit spike trains used for its calculation. We demonstrated that the theoretical association between coherence and number of spike trains is valid within small errors (Table I) and we used it for assessing the strength of common input when fatigue develops (Figures 1-3).

\section{CONCLUSION}

In this paper, a new indicator of common input strength has been introduced and applied in the context of the neurophysiological changes induced by the occurrence of fatigue. Through theoretical and experimental derivations we have reported that during fatigue the strength of the common component of the synaptic input to motor neurons increases with respect to the non-common one. This may be interpreted as a strategy used by CNS to counteract the occurrence of fatigue and the concurrent decrease in force.

\section{REFERENCES}

[1] Lemon, R. (2008). Descending Pathways in Motor Control. Ann Rev Neurosci, 31, 195-218.

[2] Farina, D., \& Negro, F. (2015). Common Synaptic Input to Motor Neurons, Motor Unit Synchronization and Force Control. Exerc Sport Sci Rev, 43(1), 23-33.

[3] Baker, S. N., Kilner, J. M., Pinches, E. M., \& Lemon, R. N. (1999). The role of synchrony and oscillations in the motor output. Exp Brain Res, 128(1-2), 109-117.

[4] Schmied, A., \& Descarreaux, M. (2010). Influence of contraction strength on single motor unit synchronous activity. Clin Neurophysiol, 121(10), 1624-1632.

[5] Baker, J. R., Davey, N. J., Ellaway, P. H., \& Fridland, C. (1992). Shortterm synchrony of motor unit discharge during weak isometric contraction in Parkinson's disease. Brain, 115(1), 137-154.

[6] Holtermann, A., Grönlund, C., Karlsson, J. S., \& Roeleveld, K. (2009). Motor unit synchronization during fatigue: described with a novel sEMG method based on large motor unit samples. J Electromyogr Kinesiol, 19(2), 232-241.
[7] Kirkwood P, and Sears T. The synaptic connexions to intercostal motoneurones as revealed by the average common excitation potential. J Physiol, 275(1), 103-134, 1978.

[8] Sears TA, and Stagg D. Short-term synchronization of intercostal motoneurone activity. J Physiol, 263(3), 357-381, 1976.

[9] Nordstrom, MA., Fuglevand, AJ., \& Enoka, RM. (1992). Estimating the strength of common input to human motoneurons from the crosscorrelogram. J Physiol, 453(1), 547-574

[10] Rosenberg JR, Amjad AM, Breeze P, Brillinger DR, and Halliday DM. (1989). The Fourier approach to the identification of functional coupling between neuronal spike trains. Progr Biophys Mol Biol, 53(1), $1-31$.

[11] De Luca, C. J., LeFever, R. S., McCue, M. P., \& Xenakis, A. P. (1982). Control scheme governing concurrently active human motor units during voluntary contractions. J Physiol, 329(1), 129-142.

[12] Vallbo, A. B., \& Wessberg, J. (1993). Organization of motor output in slow finger movements in man. J Physiol, 469(1), 673-691.

[13] Brown, P., Salenius, S., Rothwell, J. C., \& Hari, R. (1998). Cortical correlate of the Piper rhythm in humans. J Neurophysiol, 80(6), 29112917.

[14] Castronovo, A.M., Negro, F., Farina, D. (2014). The relative strength of common synaptic input to motor neurons increases with force. Bernstein Conference Göttingen, September 3-5 ${ }^{\text {th }} 2014$, pp. 130-131 doi: 10.12751/nncn.bc2014.0142, http://www.nncn.de/en/bernsteinconference/2014/abstracts/ProceedingsBC142.pdf

[15] Ushiyama J, Katsu M, Masakado,Y, et al. Muscle fatigue-induced enhancement of corticomuscular coherence following sustained submaximal isometric contraction of the Tibialis Anterior muscle. $J$ Appl Physiol, 110 (5), 1233-1240, 2011.

[16] Kattla S, and Lowery MM. (2010) Fatigue related changes in electromyographic coherence between synergistic hand muscles. Exp Brain Res, 202(1), 89-99

[17] Yang Q, Fang Y, Sun C, et al. (2009) Weakening of functional corticomuscular coupling during muscle fatigue. Brain Research, 1250, 101-112.

[18] Semmler JG, Ebert SA, and Amarasena J. (2013). Eccentric muscle damage increases intermuscular coherence during a fatiguing isometric contraction. Acta Physiol, 208(4), 362-375.

[19] Negro F., Dideriksen J., Yavuz U., Farina D (2013). Motor unit synchronization revisited: Estimation of the strength of common symaptic input to populations of motor neurons. 2013 Neuroscience Meeting Planner, San Diego, CA, http://www.abstractsonline.com/plan/start.aspx?mkey=\%7B8D2A5BE C-4825-4CD6-9439-B42BB151D1CF\%7D

[20] Sharafi, N., Benda, J., \& Lindner, B. (2013). Information filtering by synchronous spikes in a neural population. Journal of Computational Neuroscience, 34(2), 285-301.

[21] Negro F, \& Farina D. (2012). Factors influencing the estimates of correlation between motor unit activities in humans. PLOS One, 7(9), e44894.

[22] Farina D, Negro F, and Dideriksen J. (2014). The effective neural drive to muscles is the common synaptic input to motor neurons. $J$ Physiol, 592(16), 3427-3441.

[23] Holobar A, \& Zazula D. (2007). Multichannel blind source separation using convolution kernel compensation. Sig Process IEEE Trans on, $\mathbf{5 5}(9), 4487-4496$.

[24] Negro F, \& Farina D. (2011). Linear transmission of cortical oscillations to the neural drive to muscles is mediated by common projections to population of motoneurons in humans. J Physiol, 589(3), 629-637.

[25] Berg, R. W., Alaburda, A., \& Hounsgaard, J. (2007). Balanced inhibition and excitation drive spike activity in spinal half-centers. Science, 315(5810), 390-393. 